\title{
Z-TOPSIS Approach for Performance Assessment using Fuzzy Similarity
}

\author{
Ku Muhammad Naim Ku Khalif ${ }^{1} \&$ Alexander Gegov $^{2}$ \\ ${ }^{1,2}$ School of Computing, \\ University of Portsmouth, \\ PO1 3HE United Kingdom \\ \{muhammad.khalif, alexander.gegov\}@port.ac.uk
}

\author{
Ahmad Syafadhli Abu Bakar ${ }^{3}$ \\ Mathematics Division, Centre for Foundation Studies in Science, \\ Centre of Research for Computational Sciences \& Informatic Biology, Bioindustry, Environment, Agriculture and Healthcare \\ University of Malaya, Malaysia. \\ ahmadsyafadhli@um.edu.my
}

\begin{abstract}
This paper presents fuzzy similarity based Fuzzy Technique for Order Performance by Similarity to Ideal Solution (TOPSIS) for $z$-numbers. The classical fuzzy TOPSIS techniques use closeness coefficient to determine the rank order by calculating Fuzzy Positive Ideal Solution (FPIS) and Fuzzy Negative Ideal Solution (FNIS) simultaneously. The authors propose fuzzy similarity to replace closeness coefficient by doing ranking evaluation. Fuzzy similarity is used to calculate the similarity between two fuzzy ratings (FPIS and FNIS). Fuzziness is not sufficient enough when dealing with real information and a degree of reliability of the information is very critical. Hence, the implementation of $\mathrm{z}$-numbers is taken into consideration as they can capture better the knowledge of human being and are extensively used in uncertain information development to deal with linguistic decision making problems. A numerical example is given to illustrate the application of the proposed technique in ranking company performance assessment. The results show that it is highly feasible to use the proposed technique in performance assessment.
\end{abstract}

Keywords-Multi criteria decision making, fuzzy TOPSIS, fuzzy similarity, z-numbers, human intuition.

\section{INTRODUCTION}

Multiple criteria decision making (MCDM) has become a discipline of operations research which has been widely explored by experts or practitioners [1]. It is the process of making decision in the presence of multiple criteria or objectives. Nowadays, uncertainty affects strongly the world where much of the information on which decisions are based is uncertain [2], [3].

In the literature on fuzzy set, Zadeh [4] introduced fuzzy set theory in representing vagueness or imprecision in a mathematical approach. In order to do so, the main motivation of using fuzzy sets is based on its ability in appropriately dealing with imprecise numerical quantities and subjective preferences of decision makers [5]. Zadeh [6] proposed the notion of $\mathrm{z}$ number, which is an ordered pair of fuzzy numbers. The component plays the role of a fuzzy restriction and represents the information about an uncertain variable, while the component is a reliability of component and enables to represent an idea of certainty or probability [7], [8]. The idea of z-numbers is to provide a basis for computation with numbers which are not completely reliable and is more intelligent to describe the knowledge of human beings and capable to cater uncertain information.

The concept of fuzzy TOPSIS is based on the chosen alternative that should be at the shortest distance from the fuzzy positive ideal solution (FPIS) and longest distance from the fuzzy negative ideal solution (FNIS). Fuzzy TOPSIS at present offers a solution for decision makers when dealing with real world data that are usually multi criteria and involves a complex decision making process [9]. Regarding the level of interaction of with decision makers to imprecise data collection, fuzzy TOPSIS provides good agility in the decision process. In fuzzy TOPSIS, a vertex method is applied to calculate the distance between two fuzzy ratings, which calculate the distance of each alternative from FPIS and FNIS respectively using closeness coefficient. A higher value of the closeness coefficient indicates that an alternative is closer to FPIS and farther from FNIS. In this paper, fuzzy TOPSIS is modified to use fuzzy similarity [10] for ranking evaluation instead of using closeness coefficient. Fuzzy similarity is used to calculate the similarity between two fuzzy ratings.

In real world decision making problems, linguistic variables tend to be very complex to handle but they make more sense than classical fuzzy numbers. Rather than using classical fuzzy numbers, the linguistic scales are expressed in a more details and flexible way by $\mathrm{z}$-numbers. The membership function of type-1 and type-2 fuzzy sets have no information regarding knowledge of human beings. This issue has motivated the authors to propose fuzzy similarity based fuzzy TOPSIS technique that has capability to handle knowledge of human being and uncertain information properly using z-numbers. The proposed methodology is constructed without losing the generality of the fuzzy similarity and fuzzy TOPSIS in fuzzy environment. Also, it is applied for company performance assessment using znumbers. 
The rest of this paper is organized as follows: Section II introduces the concept of z-numbers. Section III presents the proposed methodology of Z - TOPSIS using fuzzy similarity. In Section VI, a numerical example is presented in implementing the $\mathrm{Z}$ - TOPSIS using fuzzy similarity for company performance. Section V summarises the conclusion.

\section{PRELIMINARIES}

In this section, we briefly review some basic concepts and definitions that are illustrated as follows.

\section{A. Z-numbers}

A z-number is an ordered pair of fuzzy numbers

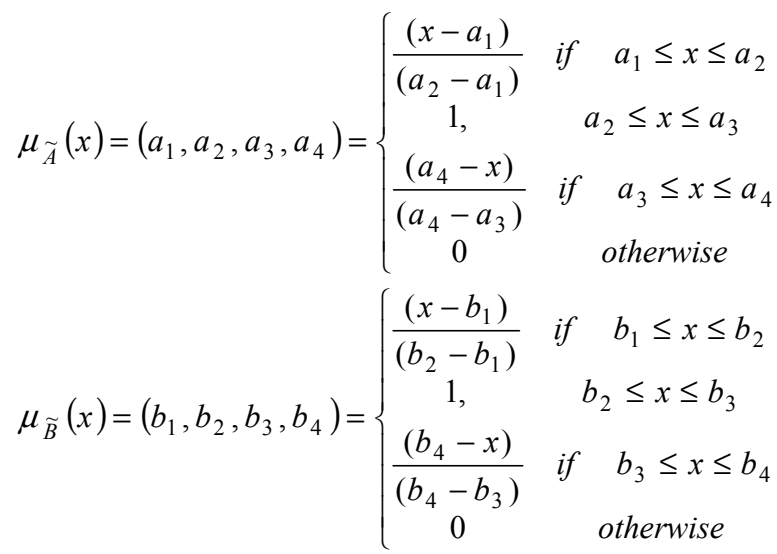

denoted as $Z=(\widetilde{A}, \widetilde{B})$. First component, $\widetilde{A}$ is known as restriction component whereby it is a real-valued uncertain on $X$ while second component, $\widetilde{B}$ is a measure of reliability for $\widetilde{A}[6]$. The illustration for z-number is depicted in Fig. 1 [7] .

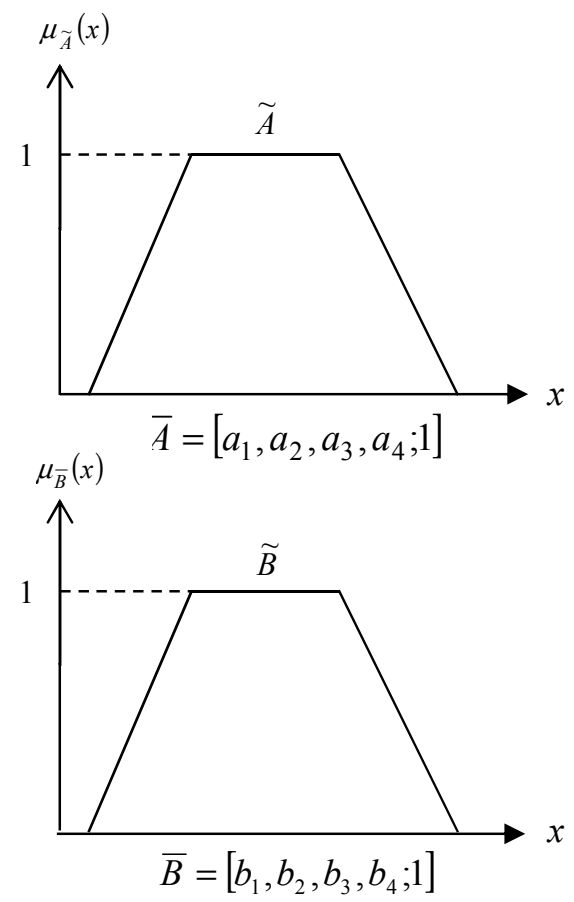

Fig. 1: Z-number, $Z=(\widetilde{A}, \widetilde{B})$

\section{Proposed Methodology}

This section focuses on the development of $Z$ - TOPSIS using fuzzy similarity. The methodology of $Z$ - TOPSIS using fuzzy similarity is extended from [11].

Step 1: Determine the weights of evaluation criteria. The weighting of evaluation criteria are employed.

\section{Step 2: Construct a hierarchy structure.}

The construction of hierarchy model shows the dependency of criteria towards alternatives that needs judgement matrix filled by decision makers about the evaluation of all criteria. Fuzzy linguistic terms are used to present the evaluation values of the alternatives preferences with respect to different criteria with degree of confidence (reliability) based on z-numbers respectively.

\section{Step 3: Construct the fuzzy decision matrix for alternatives'} evaluation.

The fuzzy decision matrix is constructed and fuzzy linguistic terms is used to evaluate the alternatives with respect to criteria.

$$
\overline{D M}=\begin{gathered}
A_{1} C_{1} \\
A_{2} \\
\vdots \\
A_{m}
\end{gathered}\left[\begin{array}{cccc}
\tilde{x}_{11} & \tilde{x}_{12} & \cdots & C_{n} \\
\tilde{x}_{21} & \tilde{x}_{22} & \cdots & \tilde{a}_{2 n} \\
\vdots & \vdots & \ddots & \vdots \\
\tilde{x}_{m 1} & \tilde{x}_{m 1} & \cdots & \tilde{x}_{m n}
\end{array}\right]
$$

where $i=1,2, \ldots, m ; j=1,2, \ldots, n$

Step 4: Convert the z-numbers into type-1 fuzzy numbers and aggregate them.

All z-numbers from fuzzy decision matrices are converted into type-1 fuzzy numbers by reduction process using intuitive vectorial centroid.

The intuitive vectorial centroid is an extension of the classical vectorial centroid methods for fuzzy numbers that proposed by [12]. Compare to other centroid methods in the literature, the way to get the centroid value is more intelligent manner, easy to compute, more balance, and consider all feasible cases of fuzzy numbers. Intuitive vectorial centroid can be computed as

$$
\operatorname{IVC}\left(\tilde{x}_{\widetilde{A}}, \tilde{y}_{\widetilde{A}}\right)=\left(\frac{2\left(a_{1}+a_{4}\right)+7\left(a_{2}+a_{3}\right)}{18}, \frac{7 h_{\widetilde{A}}}{18}\right)
$$

where

$\tilde{x}:$ the centroid point on the horizontal $x$-axis

$\tilde{y}$ : the centroid point on the vertical $y$-axis

$(\tilde{x}, \tilde{y}):$ the centroid coordinate of fuzzy number $\tilde{A}$

The reduction process of z-numbers into type-1 fuzzy sets using intuitive vectorial centroid can be computed as follows:

Assume a z-number, $Z=(\widetilde{A}, \widetilde{B})$, which is describe in Fig. 1. Let $\widetilde{A}=\left\{\left\langle x, u_{\widetilde{A}}(x)\right\rangle \mid x \in[0,1]\right\} \quad$ and $\widetilde{B}=\left\{\left\langle x, u_{\widetilde{B}}(x)\right\rangle \mid x \in[0,1]\right\}$, $u_{\widetilde{A}}(x)$ and $u_{\widetilde{B}}(x)$ are trapezoidal membership function. 
Step 1: Converting the reliability component on $x$-coordinate into crisp number or weight using intuitive vectorial centroid method from equation (3), $I V C_{\widetilde{B}}(\widetilde{x})=\frac{2\left(b_{1}+b_{4}\right)+7\left(b_{2}+b_{3}\right)}{18}=\alpha$.

Step 2: Add the weight of reliability component to the restriction component. The weighted z-number can be denoted as $\widetilde{Z}^{\alpha}=\left\{\left\langle x, \mu_{\widetilde{B}^{\alpha}}(x)\right\rangle \mid \mu_{\widetilde{B}^{\alpha}}(x)=\alpha \mu_{\widetilde{B}^{\alpha}}(x), x \in[0,1]\right\}$.

Theorem 1:

$$
E_{\widetilde{A}^{\alpha}}(x)=\alpha E_{\widetilde{A}}(x), \quad x \in X
$$

Subject to:

$$
\mu_{\widetilde{A}^{\alpha}}(x)=\alpha \mu_{\widetilde{A}^{\alpha}}(x), x \in X
$$

Proof:

$E_{\widetilde{A}^{\alpha}}(x)=\left[a_{1}, a_{2}, a_{3}, a_{4} ; \frac{2\left(b_{1}+b_{4}\right)+7\left(b_{2}+b_{3}\right)}{18}\right]=\left[a_{1}, a_{2}, a_{3}\right.$,

$\left.a_{4} ; \alpha\right]=\alpha E_{\widetilde{A}}(x)$

which can be denoted by the Fig. 3 [7] below:

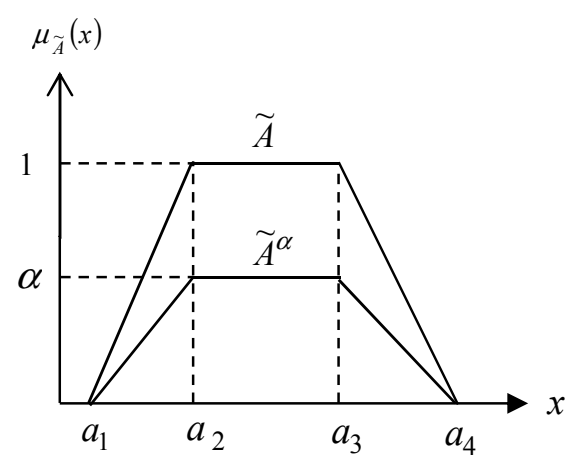

Fig. 3: Z-number after multiplying the reliability

Step 3: Convert the irregular fuzzy number (weighted restriction) to regular fuzzy number that denoted as $\widetilde{Z} \tilde{Z}^{\prime}=\left\{\left\langle x, \mu_{\widetilde{Z}^{\prime}}(x)\right\rangle \mid \mu_{\widetilde{Z}^{\prime}}(x)=\mu_{\widetilde{A}}(\sqrt{\alpha} x), x \in[0,1]\right\} . \quad$ In accordance with the Theorem 3 , the conclusion can be made that $\widetilde{Z}^{\prime}$ has the same fuzzy expectation with $\widetilde{Z}^{\alpha}$ where both are equal with fuzzy expectation.

Theorem 2:

$$
E_{\widetilde{Z}^{\prime}}(x)=\alpha E_{\widetilde{A}}(x), \quad x \in \sqrt{\alpha} X
$$

Subject to:

$$
\mu_{\widetilde{Z}^{\prime}}(x)=\mu_{\widetilde{A}}(\sqrt{\alpha} x), \quad x \in \sqrt{\alpha} X
$$

Proof:

$E_{\widetilde{Z}^{\prime}}(x)=\left[a_{1}, a_{2}, a_{3}, a_{4} ; \sqrt{\frac{2\left(b_{1}+b_{4}\right)+7\left(b_{2}+b_{3}\right)}{18}}\right]=\left[a_{1}, a_{2}, a_{3}\right.$,

$\left.a_{4} ; \sqrt{\alpha}\right]=\sqrt{\alpha} E_{\widetilde{A}}(x)$

which can be denoted by the Fig. 4 as follows [7]:

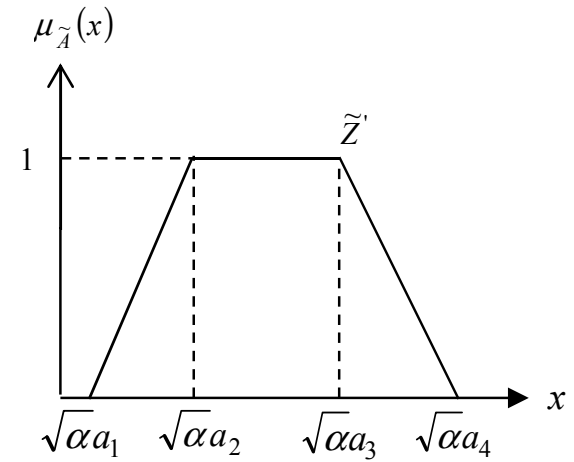

Fig. 4: The regular fuzzy number transformed from z-number

Theorem 3:

$$
E_{\widetilde{Z}^{\prime}}(x)=E_{\widetilde{A}^{\alpha}}(x)
$$

Proof:

$$
\begin{aligned}
& E_{\widetilde{A}^{\alpha}}(x)=\alpha E_{\widetilde{A}^{\prime}}(x) \\
& E_{\widetilde{Z}^{\prime}}(x)=\alpha E_{\widetilde{A}}(x) \\
& E_{\widetilde{Z}^{\prime}}(x)=E_{\widetilde{A}^{\alpha}}(x)
\end{aligned}
$$

Then, aggregate fuzzy decision matrix from decision makers' evaluation.

$$
\tilde{x}_{i j}=\left(\tilde{x}_{i j}^{1} \times \tilde{x}_{i j}^{2} \times \ldots \times \tilde{x}_{i j}^{n}\right)^{1 / k}
$$

where $\tilde{x}_{i j}$ is the performance rating of alternatives, $A_{i}$ with respect to criterion, $C_{j}$ evaluated by $k t h$ experts and $\tilde{x}_{i j}=\left(a_{1}^{k}, a_{2}^{k}, a_{3}^{k}, a_{4}^{k} ; h^{k}\right)$.

Step 5: Fuzzy decision matrix is weighted, averaged and normalised. Then, defuzzify the standardized generalised fuzzy numbers into coordinate form, $(\tilde{x}, \tilde{y})$.

The weighted fuzzy normalized decision matrix is denoted by $\widetilde{V}$ as depicted below with numerical example:

$$
\widetilde{V}=\left[\widetilde{v}_{i j}\right]_{m \otimes n} ; \quad i=1,2, \ldots, m ; \quad j=1,2, \ldots, n
$$

where

$$
\widetilde{v}_{i j}=\widetilde{x}_{i j} \times \widetilde{w}_{j}
$$

Get the average from all criteria using:

$$
\widetilde{v}_{i j}=\frac{1}{K}\left(\tilde{v}_{i j}^{1} \oplus,,, \oplus \tilde{v}_{i j}^{k} \oplus,,, \widetilde{v}_{i j}^{K}\right)
$$

Normalized each generalised trapezoidal fuzzy numbers into standardized generalised fuzzy numbers using [11]:

$$
\begin{aligned}
& a_{1_{1}}^{\prime}=\frac{a_{1}-\min \left(a_{1}, b_{1}\right)}{\max \left(a_{4}, b_{4}\right)-\min \left(a_{1}, b_{1}\right)}, \\
& a_{1_{2}}^{\prime}=\frac{a_{2}-\min \left(a_{1}, b_{1}\right)}{\max \left(a_{4}, b_{4}\right)-\min \left(a_{1}, b_{1}\right)},
\end{aligned}
$$




$$
\begin{aligned}
& a_{\prime_{3}}^{\prime}=\frac{a_{3}-\min \left(a_{1}, b_{1}\right)}{\max \left(a_{4}, b_{4}\right)-\min \left(a_{1}, b_{1}\right)}, \\
& a_{1^{\prime}}^{\prime}=\frac{a_{4}-\min \left(a_{1}, b_{1}\right)}{\max \left(a_{4}, b_{4}\right)-\min \left(a_{1}, b_{1}\right)},
\end{aligned}
$$

Step 6: Determine the fuzzy positive-ideal solution (FPIS).

Referring to normalize trapezoidal fuzzy weights, the FPIS, $A^{+}$ represents the compromise solution. The range belong to the closed interval [0,1]. The FPIS $A^{+}$(aspiration levels) is depicted as follows:

$$
A^{+}=[1,1,1,1 ; 1]
$$

The FPIS, $A^{+}$can be obtained by centroid method for $\left(x_{A^{+}}, y_{A^{+}}\right)$

\section{Step 7: Calculate the similarity of each alternative from FPIS using similarity matrix.}

Concept of TOPSIS method originally proposed by [13]. Here, the authors propose fuzzy similarity to replace closeness coefficient by doing ranking evaluation. The similarity matrix is calculated based on fuzzy similarity [10]. Determine the ranking order from values of similarity measure for all alternatives using fuzzy similarity measure proposed by [10].

$S(\widetilde{A}, \widetilde{B})=\left[1-\left|x_{\widetilde{A}}^{*}-x_{\widetilde{B}}^{*}\right|\right] \times\left[1-\left|h_{\widetilde{A}}-h_{\widetilde{B}}\right| \times \frac{\min (P(\widetilde{A}), P(\widetilde{B}))+\min (A(\widetilde{A}), A(\widetilde{B}))}{\max (P(\widetilde{A}), P(\widetilde{B}))+\max (A(\widetilde{A}), A(\widetilde{B}))}\right]$

where,$x_{\tilde{A}}^{*}$ and $x_{\widetilde{B}}^{*}$ are the horizontal centre of gravity of the generalised fuzzy numbers $\widetilde{A}$ and $\widetilde{B}$ that calculated using intuitive vectorial centroid (3).

$P(\widetilde{A})$ and $P(\widetilde{B})$ are the parameter of two generalised trapezoidal fuzzy numbers $\widetilde{A}$ and $\widetilde{B}$, calculated as follows:

$$
\begin{aligned}
& P(\tilde{A})=\sqrt{\left(a_{1}-a_{2}\right)^{2}+h_{\widetilde{A}}^{2}}+\sqrt{\left(a_{3}-a_{4}\right)^{2}+h_{\widetilde{A}}^{2}}+\left(a_{1}-a_{2}\right)+\left(a_{3}-a_{4}\right) \\
& P(\widetilde{B})=\sqrt{\left(b_{1}-b_{2}\right)^{2}+h_{\widetilde{B}}^{2}}+\sqrt{\left(b_{3}-b_{4}\right)^{2}+h_{\widetilde{B}}^{2}}+\left(b_{1}-b_{2}\right)+\left(b_{3}-b_{4}\right)
\end{aligned}
$$

$A(\widetilde{A})$ and $A(\widetilde{B})$ are the areas of two generalised trapezoidal fuzzy numbers $\tilde{A}$ and $\widetilde{B}$, calculated as follows:

$$
\begin{aligned}
& A(\widetilde{A})=\frac{1}{2} h_{\widetilde{A}}\left(a_{3}-a_{2}+a_{4}-a_{1}\right) \\
& A(\widetilde{B})=\frac{1}{2} h_{\widetilde{B}}\left(b_{3}-b_{2}+b_{4}-b_{1}\right)
\end{aligned}
$$

The larger the value of $S(\widetilde{A}, \widetilde{B})$, the more the similar between two generalised fuzzy numbers $\widetilde{A}$ and $\widetilde{B}$.

\section{NUMERICAL EXAMPLE}

A case study of company performance assessment is presented here. Two experts/ decision makers (DMs), DM1 and DM2 are used to evaluate 25 listed companies in Malaysia by market capital that make up the FTSE Bursa Malaysia KLCI (Last updated: 29 September 2016) [14]. Five criteria are considered to evaluate the companies which are: operation $\left(\mathrm{C}_{1}\right)$, marketing $\left(\mathrm{C}_{2}\right)$, customer $\left(\mathrm{C}_{3}\right)$, production $\left(\mathrm{C}_{4}\right)$ and, financial $\left(\mathrm{C}_{5}\right)$. This study simplify the concept of alternatives evaluation to $\mu \in[0,1]$ for fuzzy events. The values of alternatives evaluation A correspond to Z-numbers. The proposed Z - TOPSIS using fuzzy similarity ( $Z$ - TOPSIS - FS) is compared with $Z-A H P[8]$ and $Z$ - TOPSIS [15] from the literature for comparative study.

\section{Step 1: Determine the weights of evaluation criteria.}

The weight of evaluation criteria are employed as same value which is 0.2 for each criterion, where the total up is 1 .

\section{Step 2: Construct a hierarchy structure.}

The construction of hierarchy model shows the dependency of criteria towards alternatives as presented in Fig. 5 below.

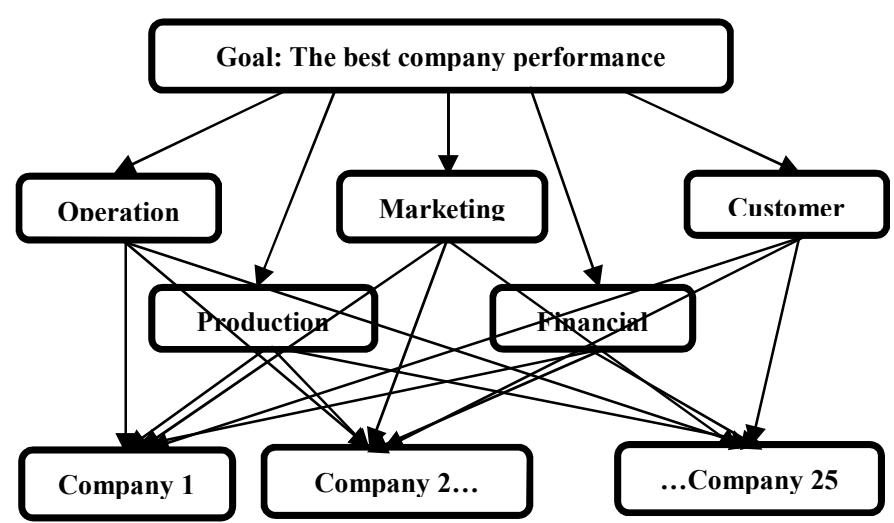

Fig. 5: The hierarchy of company performance assessment

Step 3: Construct the fuzzy decision matrix for alternatives' evaluation.

The fuzzy decision matrices are constructed and fuzzy linguistic terms from Table 1 and 2 are used to evaluate the alternatives with respect to criteria. Table 1 and 2 show the fuzzy number description used to describe the linguistic values for the restriction component and reliability component in representing z-numbers for company performance assessment by 2 decision makers.

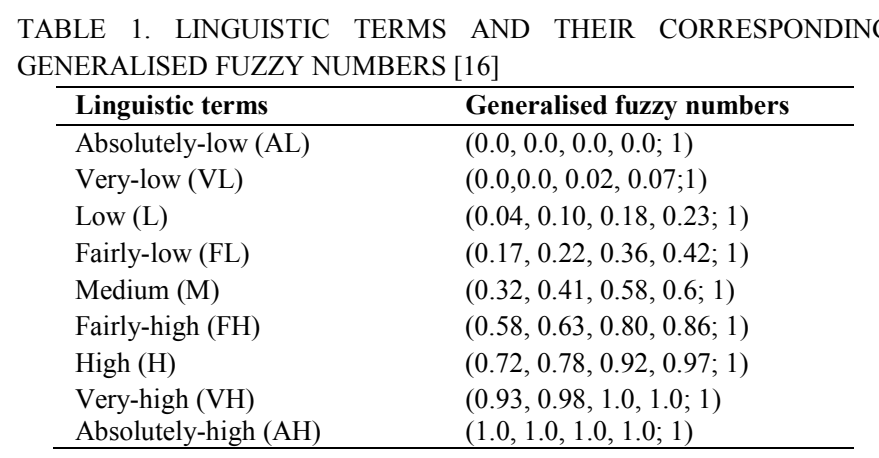


TABLE 2. RELIABILITY LINGUISTIC TERMS AND THEIR CORRESPONDING Z-NUMBERS [17]

\begin{tabular}{ll}
\hline Linguistic terms & Generalised fuzzy numbers \\
\hline Very-low (VL) & $(0,0,0,0.25 ; 1)$ \\
Low (L) & $(0,25,0.25,0.5 ; 1)$ \\
Medium (M) & $(0.25,0.5,0.5,0.75 ; 1)$ \\
High (H) & $(0.5,0.75,0.75,1 ; 1)$ \\
Very-high (VH) & $(0.75,1,1,1 ; 1)$ \\
\hline
\end{tabular}

Step 4: Convert the z-numbers into regular fuzzy numbers and aggregate the DMs' preferences.

The fuzzy decision matrices of DMs' preferences of z-numbers are converted and aggregated using equation (6-13) and (14) respectively.
Step 5: Fuzzy decision matrix is weighted, averaged and normalised.

Fuzzy decision matrix is weighted, averaged and normalised using equation (21), (22) and (23) respectively. All these results are depicted as fuzzy performance score as shown in Table 3.

\section{Step 6: Determine the fuzzy positive-ideal solution (FPIS).}

The FPIS, $A^{+}$is depicted as equation (19) and can be obtained by centroid method for $\left(x_{A^{+}}, y_{A^{+}}\right)$.

Step 7: Calculate the similarity of each alternative from FPIS using similarity matrix.

The similarity measure process is calculated using equation (25). The results of similarity measure are depicted in Table 3.

TABLE 3. THE NORMALISED AVERAGED WEIGHTED FUZZY PERFORMANCE SCORE FOR EACH COMPANY AND RANKING RESULTS BETWEEN PROPOSED TECHNIQUE AND ESTABLISHED TECHNIQUES

\begin{tabular}{|c|c|c|c|c|c|c|c|}
\hline Company & $\begin{array}{c}\text { Actual } \\
\text { rank }\end{array}$ & Fuzzy performance score & $\begin{array}{c}\text { Centroid } \\
(\mathbf{x})\end{array}$ & $\begin{array}{c}\text { Similarity } \\
\text { measure }\end{array}$ & $\begin{array}{c}\text { Z- } \\
\text { TOPSIS- } \\
\text { FS } \\
\end{array}$ & $\begin{array}{c}\mathbf{Z}-\mathbf{A H P} \\
{[8]}\end{array}$ & $\begin{array}{c}\mathbf{Z}- \\
\text { TOPSIS } \\
{[15]}\end{array}$ \\
\hline Hap Seng Consolidated Berhad & 21 & $(0.3787,0.4519,0.6701,0.7499 ; 1)$ & 0.5617 & 0.3887 & 19 & 19 & 21 \\
\hline IOI Corporation Berhad & 14 & $(0.5423,0.6155,0.8217,0.8949 ; 1)$ & 0.7186 & 0.5054 & 14 & 12 & 15 \\
\hline Sime Darby Berhad & 6 & $(0.7100,0.7818,0.9122,0.9548 ; 1)$ & 0.8438 & 0.6576 & 6 & 6 & 7 \\
\hline Kuala Lumpur Kepong Berhad & 18 & $(0.3787,0.4758,0.6701,0.7446 ; 1)$ & 0.5704 & 0.4006 & 18 & 18 & 18 \\
\hline Nestle (Malaysia) Berhad & 23 & $(0.2765,0.3583,0.5707,0.6506 ; 1)$ & 0.4643 & 0.3218 & 23 & 23 & 19 \\
\hline Petronas Dagangan Bhd & 20 & $(0.3540,0.4355,0.6369,0.7120 ; 1)$ & 0.5355 & 0.3764 & 20 & 20 & 22 \\
\hline Petronas Chemicals Group Berhad & 5 & $(0.7193,0.7951,0.9335,0.9800 ; 1)$ & 0.8610 & 0.6617 & 5 & 4 & 3 \\
\hline Axiata Group Berhad & 8 & $(0.5982,0.6754,0.8696,0.9388 ; 1)$ & 0.7716 & 0.5497 & 8 & 7 & 9 \\
\hline Petronas Gas Berhad & 7 & $(0.6262,0.6953,0.8537,0.9069 ; 1)$ & 0.7727 & 0.5805 & 7 & 8 & 6 \\
\hline YTL Corporation Berhad & 25 & $(0.0000,0.0918,0.2887,0.3710 ; 1)$ & 0.1892 & 0.1323 & 25 & 25 & 25 \\
\hline IHH Healthcare Berhad & 4 & $(0.7286,0.8018,0.9282,0.9694 ; 1)$ & 0.8614 & 0.6745 & 4 & 5 & 5 \\
\hline Hong Leong Financial Group Berhad & 24 & $(0.1407,0.2250,0.4234,0.5024 ; 1)$ & 0.3236 & 0.2274 & 24 & 24 & 24 \\
\hline PPB Group Berhad & 21 & $(0.3454,0.4279,0.6541,0.7379 ; 1)$ & 0.5412 & 0.3688 & 21 & 21 & 19 \\
\hline CIMB Group Holdings Berhad & 9 & $(0.5796,0.6554,0.8537,0.9242 ; 1)$ & 0.7539 & 0.5348 & 9 & 9 & 11 \\
\hline Telekom Malaysia Berhad & 17 & $(0.4359,0.5171,0.7313,0.8098 ; 1)$ & 0.6239 & 0.4320 & 17 & 17 & 14 \\
\hline MISC Berhad & 12 & $(0.5450,0.6262,0.8244,0.8962 ; 1)$ & 0.7242 & 0.5118 & 12 & 11 & 11 \\
\hline Public Bank Berhad & 2 & $(0.7837,0.8488,0.9057,0.9191 ; 1)$ & 0.8715 & 0.7610 & 2 & 2 & 2 \\
\hline Genting Malaysia Berhad & 16 & $(0.4732,0.5570,0.7632,0.8390 ; 1)$ & 0.6592 & 0.4602 & 16 & 16 & 15 \\
\hline Malayan Banking Berhad & 3 & $(0.7472,0.8217,0.9441,0.9840 ; 1)$ & 0.8715 & 0.6915 & 3 & 3 & 3 \\
\hline RHB Bank Berhad & 22 & $(0.3230,0.4193,0.6167,0.6903 ; 1)$ & 0.5177 & 0.3668 & 22 & 22 & 23 \\
\hline Digi.com Berhad & 10 & $(0.5796,0.6554,0.8537,0.9242 ; 1)$ & 0.7539 & 0.5348 & 10 & 9 & 11 \\
\hline Hong Leong Bank Berhad & 11 & $(0.5517,0.6222,0.8164,0.8843 ; 1)$ & 0.7190 & 0.5145 & 11 & 13 & 10 \\
\hline Genting Berhad & 15 & $(0.5048,0.5781,0.7520,0.8140 ; 1)$ & 0.6638 & 0.4864 & 15 & 15 & 7 \\
\hline Tenaga Nasional Bhd & 1 & $(0.8590,0.9282,0.9867,1.0000 ; 1)$ & 0.9512 & 0.8265 & 1 & 1 & 1 \\
\hline Maxis Berhad & 13 & $(0.5422,0.6147,0.7971,0.8621 ; 1)$ & 0.7050 & 0.5112 & 13 & 14 & 17 \\
\hline
\end{tabular}

Table 3 shows that the highest ranking result for company performance assessment is Tenaga Nasional Bhd with similarity measure value 0.8265 , while the lowest ranking is YTL Corporation Berhad with similarity measure value 0.1323 . These two companies have the highest and lowest ranking for company performance according to the actual ranking and this is in line with the ranking obtained using $Z$ - TOPSIS - FS (proposed), Z - AHP [8] and Z - TOPSIS [15]. These results show that the proposed technique is consistent with the actual ranking and other established techniques in the literature.

Comparison of the ranking results show that there are several but fairly minimal discrepancies in the ranking obtained by the three techniques and the actual ranking. Generally, most of the ranking results from these three techniques give quite similar. In fact, ranking for the Kuala Lumpur Kepong Berhad, YTL
Corporation Berhad, Hong Leong Financial Group Berhad, Public Bank Berhad, Malayan Banking Berhad and Tenaga Nasional Bhd are same for all techniques throughout. The rest of the ranking is only slightly affected. Comparing Z - AHP [8] and $\mathrm{Z}$ - TOPSIS [15], both provide inconsistent ranking results for rank 9 and 11 respectively. These duplicated ranking results present the lack of ability of Z - AHP [8] and Z - TOPSIS [15] in handling linguistic assessment properly. The proposed $Z-$ TOPSIS - FS gives prefect ordering without any duplicate ranking results. This shows that it is highly feasible to use the proposed technique in performance assessment.

Spearman's rank correlation technique is used to validate the ranking results which provides easy algebraic structure and intuitively simple interpretation. In addition, the method is less sensitive to bias due to the effect of outliers and can be used to 
reduce the weight of outliers (large distances get treated as a onerank difference). In general, the coefficient of rho, $(\rho)$ measures the strength of association between two ranked variables. The formula used to calculate Spearman's rank is shown below.

$$
\rho=1-\frac{6 \sum \partial_{i}^{2}}{n\left(n^{2}-1\right)}
$$

where

$\partial:$ the different between two ranks of each observation $n:$ the number of observations

TABLE 4. RANKING PERFORMANCE RESULTS ANALYSIS USING SPEARMAN'S RANK CORRELATION

\begin{tabular}{|c|c|c|c|}
\hline $\begin{array}{c}\text { Spearman's } \\
\text { rank }\end{array}$ & $\begin{array}{c}\mathbf{Z} \text {-TOPSIS- } \\
\text { FS }\end{array}$ & $\mathbf{Z}-\mathbf{A H P}[8]$ & $\begin{array}{c}\mathbf{Z}-\text { TOPSIS } \\
{[15]}\end{array}$ \\
\hline$\rho$ & 0.9762 & 0.9750 & 0.9146 \\
\hline
\end{tabular}

The Spearman's correlation coefficient, $\rho$ can take values between +1 to -1 . If $\rho=1$, indicates a perfect relationship of ranks, if $\rho=0$, shows no relationship between ranks and $\rho=-1$, indicates a perfect negative association of ranks. The closer $\rho$ is to zero, the weaker the relationship between the ranks. Thus based on the analysis of Spearman's rank correlation in Table 4, it is observed that the proposed Z - TOPSIS - FS outperforms the established Z - AHP [8] and Z - TOPSIS [15] from the literature.

\section{REFERENCES}

[1] A. Mardani, A. Jusoh, and E. K. Zavadskas, "Fuzzy multiple criteria decision-making techniques and applications - Two decades review from 1994 to 2014," Expert Syst. Appl., vol. 42, no. 8, pp. 4126-4148, Jan. 2015.

[2] X. Zhi-quan, "Application of Z-numbers in Multi-criteria Decision Making," in International Conference on Informantive and Cybernatics for Computational Social Systems (ICCSS), 2014, pp. 91-95.

[3] A. Gegov, F. Arabikhan, D. Sanders, B. Vatchova, and T. Vasileva, "Fuzzy networks with feedback rule bases for complex systems modelling," Int. J. Knowledge-Based Intell. Eng. Syst., 2017.

[4] L. A. Zadeh, "Fuzzy Sets-Information and Control-1965.pdf." Information and Controls, pp. 338-353, 1965.

[5] H. Deng, "Comparing and ranking fuzzy numbers using ideal solutions," Appl. Math. Model., vol. 38, no. 5-6, pp. 1638-1646, Mar. 2014.

[6] L. A. Zadeh, "A Note on Z-numbers," Inf. Sci. (Ny)., vol. 181, no. 14, pp. 2923-2932, Jul. 2011

[7] B. Kang, D. Wei, Y. Li, and Y. Deng, "A Method of Converting Znumber to Classical Fuzzy Number," J. Inf. Comput. Sci., vol. 9, no. 3, pp. 703-709, 2012.

[8] A. Azadeh, M. Saberi, N. Z. Atashbar, E. Chang, and P. Pazhoheshfar, "Z-AHP: A Z-number extension of fuzzy analytical hierarchy process," 2013 7th IEEE Int. Conf. Digit. Ecosyst. Technol., pp. 141-147, 2013.

\section{CONCLUSION}

In classical fuzzy TOPSIS, evaluation depends heavily on the selection of appropriate FPIS and FNIS by using closeness coefficient. In this paper, a fuzzy similarity method is applied to company performance selection and evaluation instead of using closeness coefficient. Rationally, replacing closeness coefficient by using fuzzy similarity measure as ranking evaluation provides better judgment for representing fuzzy numbers is many aspects (centre of gravity, parameter and area). Closeness coefficient considers the distance to both FPIS (aspiration level) and FNIS (worst level), but is does not cover the more general aspects of fuzzy numbers representation, i.e. it cannot capture the vagueness of the linguistic assessment properly. The proposed Z - TOPSIS - FS provides better selection in human based decision making problems that is capable of dealing with uncertainty in human judgment. This is helpful in situations where due to lack of access to reliable information and unavailability of complete and certain data, it is hard to make right decisions. In this sense, the consideration of z-numbers in the research work provides the use of fuzzy linguistics by considering the need of human intuition in decision making problems. As a consequence, this study presents the idea in developing to design the robust and reliable methodology for selection alternatives with respect to the resources. Hence, it can be further extended by considering more complicated case studies drawn for diverse fields of human based decision making problems.

[9] E. N. Madi and A. O. Tap, "Fuzzy TOPSIS Method in the Selection of Investment Boards by Incorporating Operational Risks," Proc. World Congr. Eng., vol. I, pp. 6-10, 2011.

[10] J. Wen, X. Fan, D. Duanmu, and D. Yong, "A modified similarity measure of generalized fuzzy numbers," Procedia Eng., vol. 15, pp. 2773-2777, 2011

[11] X. Zuo, L. Wang, and Y. Yue, "A New Similarity Measure of Generalized Trapezoidal Fuzzy Numbers and Its Application on Rotor Fault Diagnosis," Math. Probl. Eng., vol. 2013, pp. 1-10, 2013.

[12] K. M. N. Ku Khalif and A. Gegov, "Generalised Fuzzy Bayesian Network with Adaptive Vectorial Centroid," in 16th world congress of the international fuzzy systems association (IFSA) and the 9th conference of the European society for fuzzy logic and technology (EUSFLAT), 2015, pp. 757-764.

[13] C.-L. Hwang and K. Yoon, Multiple Attribute Decision Making. New York: Springer, 1981.

[14] MyPF.my, "Bursa Malaysia Top 30," 2016. [Online]. Available: http://mypf.my/investing/equities/bursa30/.

[15] R. A. Krohling and E. Santo, "TODIM and TOPSIS with Znumbers," 2016.

[16] G. Zheng, N. Zhu, Z. Tian, Y. Chen, and B. Sun, "Application of a trapezoidal fuzzy AHP method for work safety evaluation and early warning rating of hot and humid environments," Saf. Sci., vol. 50, no. 2, pp. 228-239, Feb. 2012.

[17] B. Kang, D. Wei, Y. Li, and Y. Deng, "Decision Making Using Znumbers under Uncertain Environment," J. Comput. Inf. Syst., vol. 8, no. 7, pp. 2807-2814, 2012. 